\title{
A COMPARISON OF THE EFFECTS OF AN EXPERIMENTAL METHODOLOGY FOR SPEED TRAINING ON STRENGTH AND SPEED IN ELITE SWIMMERS
}

\section{Valentin Panayotov}

\author{
National Sports Academy "Vassil Levski”, Sofia, Bulgaria
}

\begin{abstract}
Introduction: In recent years the training methodology in swimming has been following a track of fine tuning of different training styles to the specifics of elite swimming. Methodology: We used hollow rubber bands to impose a resistance, which mimics an in-water strength training. We studied 20 subjects of both sexes aged 15-18 years with at least 5 years of experience in competitive swimming. They were randomly assigned into two groups - experimental and control. The experiment was 12 weeks long. It consisted of 7 in-water and 5 dryland training sessions weekly. We modified the conventional methodology by adding 2 in-water workouts with hollow rubber bands. Maximal speed was measured by $25 \mathrm{~m}$ basic style sprint-swimming tests. Maximal strength was tested by bench pressing and bent-over rowing. Results: In the experimental group speed abilities increased by $1.9 \% \pm 1.8 \%$ and maximal strength - by $16.6 \% \pm 3.4 \%$ and $16.7 \% \pm 5.2 \%$ for the 2 test exercises respectively (mean \pm standard deviation, $p \leq 0.05$ ). No statistically significant changes were measured in the control group in speed testing. The differences in strength tests were statistically significant but smaller than those in the experimental group. Discussion: Both conventional and experimental methodologies increased maximal strength, but only the experimental succeeded in transferring strength into speed. Conclusion: The improvement in strength was much bigger than that in speed (in percentage terms). In our opinion, further investigations are necessary for estimating the exact relationships between strength and speed in elite swimmers.
\end{abstract}

Key words: swimmers, strength, speed, hollow rubber bands

\section{INTRODUCTION}

In recent years training methodology in swimming evolved and follows a trend of fine-tuning of different training protocols to the specifics of elite swimming. More precisely, the ratio of specific training means to the total training volume increased dramatically (Hines, 2008; Konstantinov, 2001; Kutinchev, 1967; Kutinchev, 1969; Platonov, Vaicehovski, 1985). Exercises on special strength and speed training machines are routinely incorporated into preparation programmes of elite swimmers (Schneider, 2017; Heazlewood, 2006; Stanula, et al., 2012; Graef et al., 2010). Different types of resistance training equipment are widely used for improving coordination structure of the exercises and attempting to mimic successfully the competitive movements. Those methodological approaches improve enormously the efficiency of strength and speed training in swimming (Platonov, Vaicehovski, 1985; Stoichev, 1977; Stanula, et al., 2012). In our study, we used hollow rubber bands to impose resistance, which to mimic an in-water strength training and to potentiate a transfer of strength into speed and power.
Aim and objectives of the study

The study aimed to test and compare the efficiency of an experimental methodology for speed training to that of the conventionally used training program for improving absolute speed and strength in elite swimmers.

The objectives of the study were as follows:

1. To recruit 20 competitive swimmers (according to the methodological guidelines of the experiment), which to be randomly assigned into two groups - one experimental and one control;

2. The participants of the experimental group to follow an experimental 12-week long methodology for speed training for elite swimmers developed by our team;

3. The participants of the control group to follow a conventional 12-week long speed training methodology for elite swimmers;

4. To test the speed and strength abilities of the participants at the beginning and the end of the experiment;

5. To measure post-hoc within-group differences in strength and speed for both groups of participants. 


\section{METHODS}

We used hollow rubber bands to impose a resistance, which to mimic an in-water strength training. We studied 20 subjects of both sexes aged 15-18 years with at least 5 years of experience in competitive swimming. They were randomly assigned into two groups - one experimental and one control. The experiment was 12 weeks long. It consisted of 7 in-water and 5 dryland training sessions weekly. We modified the conventional methodology by adding 2 in-water workouts with hollow rubber bands. Accordingly, about $30 \%$ of the volume of the experimental program consisted of highly intensive speed-strength in-water exercises with rub- ber bands. Maximal speed was measured by $25 \mathrm{~m}$ basic style sprint-swimming tests. We measured maximal strength by 2 resistance exercises - bench press and bent-over rows. The protocol consisted of 2-5 warm-up sets (using the "Pyramid" method) until the intensity of 2-3 RM was reached. Maximal strength was estimated by adding $2.5 \mathrm{~kg}$ to the working load in every consecutive set until failure. We used the free software environment $\mathrm{R}$ for processing our raw data (Wickham, Grolemund, 2017).

\section{RESULTS}

The baseline values of the studied variables are shown in Table 1.

Table 1. Baseline values of the results in 25-metre speed swimming test

\begin{tabular}{|c|c|c|}
\hline Group & Variable & Value (sec) \\
\hline $\mathbf{E}$ & Mean & 13.33 \\
\hline $\mathbf{E}$ & Standard error & 0.54 \\
\hline $\mathbf{E}$ & Standard deviation & 1.71 \\
\hline $\mathbf{C}$ & Mean & 13.67 \\
\hline $\mathbf{C}$ & Standard error & 0.65 \\
\hline $\mathbf{C}$ & Standard deviation & 2.07 \\
\hline
\end{tabular}

The standard t-test methodology was used for lis, 2002). No statistically significant between-group measuring within- and between-group differences differences were observed in baseline results of the (Freedman et al., 1980; Mendenhall, 1999; Papou- tests (see Tables 2 and 3).

Table 2. Baseline between-group t-test for differences in 25-meter speed swimming

\begin{tabular}{|c|c|c|c|c|}
\hline & Mean & Deg. of Freedom & t & $\boldsymbol{p}$ \\
\hline Value & 0.348 & 18 & 0.410 & 0.687 \\
\hline
\end{tabular}

Table 3. Baseline between-group t-test for differences in strength tests

\begin{tabular}{|c|c|c|c|c|}
\hline & Mean & Deg. of Freedom & $\mathbf{t}$ & $\mathbf{p}$ \\
\hline Bench press & -7.5 & 18 & 0.272 & 0.277 \\
\hline Bent-over rows & 6.5 & 18 & -0.769 & 0.453 \\
\hline
\end{tabular}

We found statistically significant post-hoc increas- were statistically significant only in the experimenes in muscle strength for both groups - tables 4 and tal group - tables 6 and 7.

5. The post-hoc differences in swimming speed

Table 4. Post-hoc within-group t-test for differences of the experimental group in strength tests

\begin{tabular}{|c|c|c|c|c|}
\hline & Mean & Deg. of Freedom & $\mathbf{t}$ & $\mathbf{p}$ \\
\hline Bench press & 9.5 & 9 & 15.234 & $<0.0001$ \\
\hline Bent-over rows & 9.28 & 9 & 10.088 & $<0.0001$ \\
\hline
\end{tabular}

Table 5. Post-hoc within-group t-test for differences of the control group in strength tests

\begin{tabular}{|c|c|c|c|c|}
\hline & Mean & Deg. of Freedom & $\mathbf{t}$ & $\mathbf{p}$ \\
\hline Bench press & 7.25 & 9 & 10.474 & $<0.0001$ \\
\hline Bent-over rows & 6.55 & 9 & 9.678 & $<0.0001$ \\
\hline
\end{tabular}


Table 6. Post-hoc within-group $t$-test for differences of the experimental group in 25-meter speed swimming test

\begin{tabular}{|c|c|c|c|c|}
\hline & Mean & Deg. of Freedom & $\mathbf{t}$ & $\boldsymbol{p}$ \\
\hline Value & 0.262 & 9 & 3.327 & 0.009 \\
\hline
\end{tabular}

Table 7. Post-hoc within-group t-test for differences of the control group in 25-meter speed swimming test

\begin{tabular}{|c|c|c|c|c|}
\hline & Mean & Deg. of Freedom & $\mathbf{t}$ & $\boldsymbol{p}$ \\
\hline Value & 0.048 & 9 & 1.833 & 0.100 \\
\hline
\end{tabular}

\section{DISCUSSION}

Strength training is applied in many sports as a performance-enhancing tool. A positive relationship exists between muscle strength and velocity of movement of the body (Zatsiorsky, Kraemer, 2006; Fleck \& Kraemer, 1997). Accordingly, the goal of strength training in sprint-swimming is achieving a transfer of strength to speed. In our experiment, both the conventional and the experimental methodologies increased maximal strength, but only the latter succeeded in transferring strength into speed. Interestingly, the improvement in strength was of much greater magnitude than that in speed (in relative terms) $-16.6 \% \pm 3.4 \%$ and $16.7 \% \pm 5.2 \%$ respectively for the 2 exercises we used vs. $1.9 \% \pm 1.8 \%$ for the in-water speed test. Probably, a non-linear quantitative relationship exists between strength and speed in sprint-swimming. In our opinion, specific strength training should dominate over general resistance training into preparation programs of elite swimmers. The specifics of training and competition environment in swimming hinder a successful transfer of dryland muscle strength increases into specific in-water strength and speed. Probably, the process of transition of muscle strength into swimming speed depends on a tipping point - a certain amount of additional muscle strength should be accumulated before improvements in speed to emerge. Experiments with larger sample sizes are necessary for testing those preliminary conclusions. In our opinion, further investigations are needed for quantifying the exact relations between strength and speed in elite swimmers.

\section{ACKNOWLEDGEMENTS}

The author thanks his colleagues, assistant prof. Bogomil Angelov of NSA, Sofia, Bulgaria and swimming coach Lubomir Lazarov, of SC Olympia, Sofia, Bulgaria who were responsible for the raw data collection and provided assistance and ex- pertise that greatly contributed to the research.

\section{REFERENCES}

Fleck, SJ., Kraemer, WJ. (1997). Designing Resistance Training Programs. Human Kinetics, USA

Freedman, D., Pisani, R., Purves, R. (1980). Statistics. W. W. Norton \& Company, Ney York

Graef, F., Pinto, R., Alberton, C., de Lima, W., Luiz, F. (2010). The Effects of Resistance Training Performed in Water on Muscle Strength in the Elderly, Journal of Strength and Conditioning Research: November 2010 Volume 24 - Issue 11 pp. 3150-3156

Hines, E. (2008). Fitness swimming. Human Kinetics, USA

Konstantinov, Hr. (2001), Pluvniat sport v Bulgaria prez $X X v e k$, NSA Pres, Sofia

Kutinchev, M. (1967). Za sastoianieto na pluvnia sport u nas, VFK, No. 6, pp. 25-29

Kutinchev, M. (1969). Kam nov podem $v$ razvitieto na pluvnia sport. VFK, No. 6, pp. 58-63

Mendenhall, W., Beaver, RJ., Beaver, BM. (1999). Introduction to Probability and Statistics. Duxbury Press, USA Papoulis, A. (2002). Probability, Random Variables and Stochastic Processes, McGraw Hill, USA

Platonov, V., Vaicehovski, S. (1985). Trenirovkata na visokokalificirani pluvci, Moskva

Schneider, T. (2017). Swimmer's Workout Handbook, Hatherleight Press, USA

Stoichev, A., Brogli, Ia., Boichev, Kl., Hadjiev, N., Conev, St. (1977). Prognoza za razvitieto na lekata atletika, pluvaneto i sportnata gimnastika v NRB 1971-1990 god., MF, Sofia

Wickham, H., Grolemund, G. (2017). R for data science, O'Reilly Media Inc., USA

Zatsiorsky, V., Kraemer, W. (2006). Science and practice of strength training, $2^{\text {nd }}$ ed., Human Kinetics, USA 